BULL. AUSTRAL. MATH. SOC.

$16 A 30$

VOL. II (1974), 359-364.

$(16 A 50)$

\title{
A note on regular modules
}

\section{V.S. Ramamurthi}

Kaplansky's observation, namely, a commutative ring $R$ is (von Neumann) regular if and only if each simple $R$-module is injective, is generalized to projective modules over a commutative ring.

It is a well-known observation due to Kaplansky that a commutative ring $R$ is (von Neumann) regular if and only if every simple epi-image of $R$ is injective. One may ask whether this can be extended to projective modules over arbitrary commutative rings. To be precise, call a module $P$ over a ring $R$

(i) a regular module if, for each $p \in P$, there is $f \in \operatorname{hom}_{R}(P, R)$ such that $p=p(f p)$ (Zelmanowitz [10]) and

(ii) a V-module if each simple epi-image of $P$, when it exists, is injective.

Then the question is: is a projective module $P$ over a commutative ring $R$ a regular module if and only if it is a V-module? In [9] Ware proved the implication 'only if' for all $P$ and the implication 'if' for finitely generated $P$, leaving unsettled the validity of 'if' for arbitrary $P$. This problem was solved by S. Alamelu (private commication). In this note we formulate this problem in a more general setting and prove the validity of 'if' for all $P$. We call a module $P$ over a ring $R$

(i) a weakly regular module, if for each $p \in P$, there are $f_{1}, \ldots, f_{n} \in \operatorname{hom}_{R}(P, R)$ and $r_{1}, \ldots, r_{n} \in R$ such that

Received 3 July 1974. Communicated by K.M. Rangaswand. 


$$
p=p \sum_{1}^{n} f_{i}\left(p r_{i}\right) \text {, and }
$$

(ii) an $B V$-module if every simple image of every cyclic submodule of $P$ is infective.

We show that any projective $H V$-module is weakly regular. For commutative $R$, this yields the desired result; namely, any projective $V$-module is regular.

Throughout this paper $R$ will denote an associative ring with identity and all modules considered will be unitary right $R$-modules. Homomorphisms will be written opposite to scalars. For any module $M, M^{*}$ will denote $\operatorname{hom}_{R}(M, R)$, and for any $m \in M$,

$$
T_{m}=\left\{\sum_{l}^{n} f_{i}\left(m r_{i}\right) ; f_{i} \in M^{*}, r_{i} \in R\right\}
$$

Note that $T_{m}$ is a two-sided ideal of $R$.

DEFINITION 1. An $R$-module $M$ is said to be

(i) regular, if, for every $m \in M$, there is $f \in M^{*}$ with $m=m(f m) \quad(c f .[10])$;

(ii) weakly regular if, for every $m \in M, m \in m T_{m}$;

(iii) a V-module if each simple epi-image of $M$ (when it exists) is injective;

(iv) an $H V$-moduze if each cyclic submodule of $M$ is a $V$-module.

EXAMPLES. (i) If $R$ is a simple ring with identity and $I$ is a right ideal of $R$, then $I$ is a weakly regular module. For, if $a \in I$, then $a=a \cdot 1=a \sum r_{i}^{a s}{ }_{i}\left(r_{i}, s_{i} \in R\right)$ and clearly the $r_{i}$ can be considered as elements of $I^{*}$. More generally, any right ideal of a weakly regular ring is a weakly regular module. ( $R$ is called a weakly regular ring if $a \in a R a R$ for every $a \in R$. For information on these rings, see [6].)

(ii) Any regular module is weakly regular. The converse is not true. 
For instance, if $R$ is a simple integral domain which is not a division ring (for an example of such a ring see [5], p. 211), then any right ideal of $R$ is weakly regular but not regular.

(iii) A ring $R$ has been called a V-ring, if each simple right $R$-module is injective (see [3], [4] for properties and examples of such rings). It is clear that any module over a $V$-ring is an $H V$-module.

(iv) Any $H V$-module is a $V$-module. The converse is not true (see Remark 3 (ii) below).

PROPOSITION 2. Any projective HV-module is weakly regular.

Proof. Let $P$ be a projective $H V$-module and $p \in P$. If $p \notin p^{T} p$, choose a submodule $X$ of $P$ maximal with respect to $p \notin X, p T_{p} \subset X$. Then $Y=(p R+X) / X \simeq p R / p R n X$ is a simple $R$-module and as it is an epiimage of $p R \subset P, Y$ is injective, by hypothesis. But $Y$ is an essential submodule of $P / X$ so that $Y=P / X$. Now consider the diagram

$$
\lg _{R \rightarrow P / X} \underset{f}{\lg }(p R+X) / X,
$$

where $g$ is the projection map and $f$ is the canonical map obtained by using the fact that $P / X$ is simple. As $P$ is projective, this diagram yields a map $h: P \rightarrow R$ with $f h=g$. In particular,

$$
p+X=g p=(f h) p=(f I)(h p),
$$

and if $f \mathcal{l}=p r+X$, then this gives $p-(p r)(h p) \in X$. But $(p r)(h p) \in p T_{p}$. Thus $p \in X$, a contradiction.

REMARKS 3. (i) A projective weakly regular module need not be an $H V$-module. This follows from the fact that there are (von Neumann) regular rings which are not V-rings (see [4]).

(ii) A projective $V$-module need not be weakly regular. To see this, let $R$ be a non-semi-simple artinian, right hereditary ring such that the injective hull $E$ of the right $R$-module $R$ is projective. Then $R$ is a semi-primary $Q F-3$ ring and $E$ is projective ( $c f$. Colby and Rutter [2]). As $R$ is hereditary, $E$ is a projective $V$-module, and if it is a weakly regular module, then $R$ (being a submodule of $E$ ) will be a weakly 
regular module over itself. In other words, $R$ will be a weakly regular ring and consequently its Jacobson radical will be equal to zero [6]. Since $R$ is already semi-primary, this will imply that $R$ is semi-simple artinian, contradicting our assumption that $R$ is not so. Thus $E$ is not a weakly regular module. By Proposition 2, it also follows that $E$ is not an $H V$-module.

(iii) The above proposition generalizes to projective modules the fact that any $V$-ring is a weakly regular ring; proved in [7].

(iv) A more detailed study of weakly regular modules will be presented in a subsequent paper.

THEOREM 4. If $R$ is a commutative ring, then the following are equivalent on any projective $R$-module $P$ :

(i) $P$ is weakly regular;

(ii) $P$ is reguzar;

(iii) $P$ is a V-module;

(iv) $P$ is an HV-moduze.

Prcof. We give a cyclic proof.

$(i) \Rightarrow(i i)$. Let $p \in P$. Then $p=p \sum f_{i}\left(p r_{i}\right)$ where $f_{i} \in P^{*}$ and $r_{i} \in R$. But

$$
\sum f_{i}\left(p r_{i}\right)=\sum\left(f_{i} p\right) r_{i}=\sum r_{i}\left(f_{i} p\right)=\sum\left(r_{i} f_{i}\right) p=\left(\sum r_{i} f_{i}\right) p
$$

Thus $p=p(f p)$ where $f=\sum r_{i} f_{i} \in P^{*}$ and hence $P$ is regular.

$(i i) \Rightarrow$ (iii) has been proved by Ware ([9], Proposition 2.5).

(iii) $\Rightarrow(i v)$. Let $p \in P$. As $p$ is projective, $p=\sum p_{i}\left(f_{i} p\right)$ for $p_{i} \in P, f_{i} \in P^{*}$. Let $m_{i}=f_{i} p$ and

$$
T=\left\{\sum f(\bar{p}), f \in P^{*} \text { and } \bar{p} \in P\right\}=\text { trace ideal of } P \text { in } R \text {. }
$$

It is well known (and easily proved by considering localizations of $P$ and $T$ at maximal ideals) that $R / T$ is a flat module (see, for instance, [8]). Hence, by Proposition 2.2 of [1], there is a morphism $f$ from $R$ to $T$ 
such that $f\left(m_{i}\right)=m_{i}$ for each $i$. Writing $f(1)=t$, we have $m_{i}=m_{i} t$ for each $i$ and hence $p=p t$. Hence, if $t=\sum_{1}^{n} g_{i} x_{i}$ for some $g_{i} \in P^{*}, x_{i} \in P$, then $\left(q_{i}\right) \mapsto p\left(\sum g_{i} q_{i}\right)$ defines an epimorphism from $P \oplus \ldots \oplus P$ ( $n$ copies $)$ to $p R$. It follows from this, that any simple epi-image of $p R$ is also an epi-image of $P$ and hence is injective. Thus $P$ is an $H V$-module.

(iv) $\Rightarrow$ (i) follows from Proposition 2 above.

This completes the proof.

Note added in proof (11 November 1974). It seems worthwhile noting explicitly the following result, which is a consequence of the proofs given in this paper.

Let $R$ be a (not necessarily commutative) ring with identity, and let $P$ be a projective V-module with trace ideal $T$. Then the following are equivalent:

(i)' $R / T$ is left $R$-flat;

(ii)' $P$ is an HV-module;

(iii)' $P$ is a weakly regular module.

The proof of $(i i i) \Rightarrow$ (iv) of Theorem 4 actually establishes $(i)^{\prime} \Rightarrow(i i)^{\prime}$ here. (ii)' $\Rightarrow$ (iii)' is Proposition 2. (iii)' $\Rightarrow$ (i)' follows by using the dual basis property of projective modules. Note that if $R$ is commutative, then ( $i)^{\prime}$ holds for all projectives $P$.

\section{References}

[1] Stephen U. Chase, "Direct products of modules", Trans. Amer. Math. Soc. 97 (1960), 457-473.

[2] R.R. Colby and E.A. Rutter, Jr., "Generalizations of QF-3 algebras", Trans. Amer. Math. Soc. 153 (1971), 371-386.

[3] John H. Cozzens, "Homological properties of the ring of differential polynomials", Bulz. Amer. Math. Soc. 76 (1970), 75-79. 
[4] Carl Faith, Lectures on injective modules and quotient rings (Lecture Notes in Mathematics, 49. Springer-Verlag, Berlin, Heidelberg, New York, 1967).

[5] Nathan Jacobson, Structure of rings (Colloquium Publ. 37, revised edition. Amer. Math. Soc., Providence, Rhode Island, 1964).

[6] V.S. Ramamurthl, "Weakly regular rings", Conad. Math. Bull. 16 (1973), 317-321.

[7] V.S. Ramamurthl and K.M. Rangaswamy, "Generalized V-rings", Math. Scand. 31 (1972), 69-77.

[8] Wolmer V. Vasconcelos, "On projective modules of finite rank", Proc. Amer. Math. Soc. 22 (1969), 430-433.

[9] Roger Ware, "Endomorphism rings of projective modules", Trans. Amer. Math. Soc. 155 (1971), 233-256.

[10] J. Zelmanowitz, "Regular modules", Trans. Amer. Math. Soc. 163 $(1972), 341-355$.

Arul Anandar College,

Karumathur, via Madurai,

Taml I Nadu,

India. 